\title{
I Projektdesign und Kontext
}

\section{Einleitung}

Die Herausforderungen der Inklusion der Geflüchteten, welche während der sogenannten „,refugee crisis“ von 2015/16 nach Österreich migrierten, überschneiden sich gegenwärtig mit der SARS-CoV2-Pandemie und deren Auswirkungen (ILO June 2020, 2 ff.; WORLD BANK GROUP 19-06-2020). Die Integration und Inklusion von Geflüchteten war bereits in Zeiten vor SARS-CoV2 in allen EU-Staaten ein komplexer und multidimensionaler Prozess. Seither hat die Pandemie soziale, ökonomische, gesundheits- sowie genderbezogene Ungleichheiten verstärkt (GUADAGNo 2020). Diese Disparitäten sind bei Menschen mit Fluchthintergrund besonders ausgeprägt und stellen längerfristige, nicht akzeptable Benachteiligungen dieser Gruppen in den europäischen Aufnahmegesellschaften dar (KLUGE et al. 2020).

Trotz des starken zahlenmäßigen Anstiegs der Bevölkerung mit Fluchthintergrund seit 2015 besteht nicht nur in Österreich nach wie vor ein Defizit an Forschung über diese sowie die durch SARS-CoV2 bedingten Problemkonstellationen (GAGE 2020; Hargreaves, Zenner et al. 2020; KLuge et al. 2020; The LANCet 2020).

Generell ist über die spezifischen Probleme von Personen, die eine Flucht hinter sich haben, weit weniger als über Arbeitsmigrant/inn/en bekannt (vgl. ColLINS 2020; CTV News 18-05-2020; D‘Ignoti 16-03-2020; Guadagno 2020; IMREF 2020; IOM 2020; Jordan 18-03-2020; LiEM ET AL. 2020; MaYs \& NeWMan 08-04-2020; Morgan \& Rose 2020; NASR 30-07-2020). Im Vergleich zu Arbeitsmigrant/inn/en (z.B. aus Serbien oder der Türkei), aber auch zu diversen Gruppen mit Fluchthintergrund (z.B. aus Bosnien-Herzegowina) wurde bislang in Österreich über die in rezenter Zeit durch Flucht ins Land gekommenen Menschen nur wenig geforscht.

International sind bereits im Jahr 2020 erste Studien publiziert worden, die die Situation von Covid-19 im Kontext von Flucht untersuchten (KASSEM 2020 über die Situation in Syrien, Razum 2020 über die Situation in Deutschland). Die menschenunwürdigen Lebensbedingungen und die fehlende Gesundheitsversorgung für Menschen auf der Flucht führen zu humanitären Krisen ungeahnten Ausmaßes (VoneN et al. 2020). In verschiedenen Aufnahme- und Transitländern haben Flüchtlinge kaum oder nur eingeschränkten Zugang zum öffentlichen Gesundheitssystem (SALMANI 2020 für afghanische Flüchtlinge im Iran). Bereits bestehende Risikofaktoren werden durch die Pandemie weiter verstärkt (LoPez-Pena 2020), hinzu kommt, dass Menschen auf der Flucht noch mehr stigmatisiert werden (LogIE 2020). 
Infolge der multiplen Belastungen von Flucht und Pandemie wurden Fragen der psychischen Gesundheit diskutiert, wie z.B. von EL-Kнатів et al. für die allgemeine und die Flüchtlingsbevölkerung in Jordanien (2020). Ein besonderer Fokus wurde dabei auf die Situation von Kindern und Jugendlichen gelegt (ENDALE et al. 2020; FEGERT et al. 2020). Faktoren, die die psychische Gesundheit verschlechtern, wurden sowohl mit qualitativen als auch mit quantitativen Methoden untersucht und gezeigt, wie erhöhte Angst und Unsicherheit zu psychischen Erkrankungen führen können.

Einige Studien haben die Situation von besonders vulnerablen Menschen aufgezeigt, z. B. von Personen auf der Flucht, die an chronischen Krankheiten leiden (FAWAD et al. 2020), oder die Situation älterer Menschen (PEPRAH 2020); diese Studien haben auch das Vulnerabilitätsparadigma in den von humanitären Krisen betroffenen Orten kritisch diskutiert (NoтT 2020).

Kritisch hinterfragt werden staatliche Maßnahmen wie Containment Policies (HARgreaves, Kumar et al. 2020), strukturelle Herausforderungen und Benachteiligungen in Integrationskontexten, etwa bei der ökonomischen Inklusion (DEMPSTER et al. 2020) oder in der Erwachsenenbildung (GAGE 2020). In der Konzeption von Unterstützungsmaßnahmen müssen die bereits bestehenden Initiativen der Betroffenen, d.h. deren Agency, anerkannt, unterstützt und verstärkt werden (Alıo et al. 2020).

In diesem Forschungsbereich wurde die Notwendigkeit multidisziplinärer Forschung betont (MoRGAN \& Rose 2020). So sind auch Ansätze aus sozialarbeiterischer (NiSANCI et al. 2020 zur Situation in der Türkei) oder aus ökonomischer Sicht (Peters 2020) in die Diskussion mit eingebracht worden.

Während Refugee Studies an sich schon ein spezifisches methodisches Instrumentarium und sensible Zugänge erfordern, sind in dieser Krise zusätzliche ethische Fragen evident (CALIA et al. 2020). Mehrere der angeführten Forschungen sind Panelstudien, die auf quantitative Daten oder alternativen Zugänge, wie z.B. neue Medien oder telefonische Datenerhebungen, zurückgreifen (CHEN et al. 2020). Qualitative Studien mit einer umfassenden Datenerhebung und mit einem vergleichenden Ansatz sind erst im Entstehen.

Während ein überwiegender Teil dieser ersten Studien auf die schwierige Lage von Menschen auf der Flucht, in Flüchtlingslagern oder in Transitländern fokussiert, gibt es wenige Untersuchungen, die auf die Situation von Personen Bezug nehmen, die bereits seit einigen Jahren einen gesicherten Rechtsstatus besitzen und sich eine neue Zukunftsperspektive aufgebaut haben.

Durch die vom WWTF im COVID-19 Rapid Response Call ermöglichten Forschungen in verschiedenen Disziplinen konnte auch der Fokus auf die spezifische Situation von Menschen gelegt werden, die in den letzten Jahren nach Österreich geflüchtet sind. Das von Mai bis November 2020 durchgeführte sozialwissenschaftliche Projekt ist als eine Momentaufnahme zu verstehen und zeigt, welche Auswirkungen die Pandemie und der ersten Lockdown gerade auf jene Menschen hatten, die sich in unterschiedlichen Phasen von strukturellen Integrationsprozessen (Arbeit, Wohnsi- 
tuation, Ausbildung) befanden. Der Erhebungsschwerpunkt lag auf Wien. Infolge der relativ kurzen Zeitspanne der Erhebung und der begrenzten Ressourcen konnten in die Befragung keine Vergleichssamples mit Menschen, die keine Flucht hinter sich hatten, aufgenommen werden.

Mit der Kompilation dieses Manuskripts (April 2021) während des dritten Lockdowns hat sich bereits eine Reihe von Veränderungen ergeben, die wir in diese Publikation nicht mehr aufnehmen konnten. Auch basieren die Empfehlungen für die Good-Practice-Maßnahmen auf den Erhebungen im ersten Lockdown. Es sind in den Forschungsergebnissen allerdings Tendenzen ablesbar, die die Annahme zulassen, dass sich problematische Entwicklungen nicht verbesserten, sondern sogar weiter verschärften.

Die Fokussierung auf Afghan/inn/en und Syrer/inn/en im Rahmen des Projekts wurde beispielhaft für die verschiedenen in Wien ansässigen Menschen, die nach Österreich geflüchtet sind, gewählt. Nach dem Herkunftsland betrachtet, sind dies auch die zahlenmäßig größten Gruppen. Ein Kontaktdatenpool aus mehreren vorangegangenen Studien und Kooperationsbeziehungen zu Vereinen und Organisationen aus beiden Herkunftsgesellschaften war vorhanden und ermöglichte einen Community Based Participatory Approach. Auf diese Weise konnte bei diesem hinsichtlich seines Zeitrahmens sehr knapp kalkulierten Projekt eine Optimierung der Zeitressourcen erreicht werden. Des Weiteren ermöglichte diese Auswahl die Darstellung und den Vergleich von mehrfachen Heterogenitäten (Alter, Geschlecht, Familien- und Bildungsstand etc.) und daher auch das Aufzeigen von Bereichen, die strukturelle, sozial- und gesundheitspolitische Maßnahmen erfordern.

Wir verwenden den Begriff „Communities“ dann, wenn wir ausdrücken wollen, dass es eine gewisse Gemeinsamkeit zwischen den sehr heterogenen Herkunftsgesellschaften gibt. Gemeinsamkeiten resultieren aus dem rechtlichen Status, den Strategien in der Asylpolitik bezüglich der Behandlung und (Nicht-)Gewährung von Asylanträgen von Menschen aus bestimmten Herkunftsländern oder aufgrund struktureller Bedingungen. Mit der Verwendung des Begriffs soll auf keinen Fall einer Homogenisierung Vorschub geleistet werden, sondern ganz im Gegenteil, es gilt die Diversität in politischen, ethnischen, religiösen, demographischen, strukturellen etc. Belangen ausreichend zu berücksichtigen.

Gerade in den Jahren seit 2015 und vor COVID-19 fand eine Reihe von Organisationsbildungsprozessen in Form von Vereinen wie auch virtuell statt, die auf die jetzige Situation umfangreiche Auswirkungen haben. Es erscheint uns auch diesbezüglich ein vorsichtiges Operieren mit dem Begriff sinnvoll.

Auch versuchen wir Begrifflichkeiten und Kategorisierungen zu vermeiden, die zur Flucht gezwungene Menschen als eine homogenisierte Gruppe darstellen. Wir verwenden zwar den im wissenschaftlichen Diskurs häufig kritisierten Terminus der „Integration“, obwohl wir uns dessen Problematik und der teils sehr unterschiedlichen Definitionen völlig bewusst sind. „Integration“ und „Inklusion“ sind zwei Begriffe, 
die im Zusammenhang mit Migration häufig als Auslöser von ideologisch fundierten, kontroversiellen Debatten fungieren. Nicht selten werden sie synonym benutzt, viele Wissenschaftler/innen wie auch Praktiker/innen orten allerdings erhebliche Differenzen. Der Fokus der Arbeit liegt auf der Systemintegration, d.h. auf dem Wohnungs-, Arbeits- und Bildungsbereich, und da es sich hinichtlich eines Outputs des Projekts aber um eine praxisorientierte Kompilation von Handlungsempfehlungen handelt, halten wir die Verwendung eines weit gefassten und kritisch angewendeten Integrationsbegriffs für angebracht.

\section{Projektziele}

Das generelle Projektziel lag darin, die komplexen Auswirkungen der SARSCoV2-Pandemie auf Personen, die aus Afghanistan und Syrien geflüchtet sind, in Wien zu untersuchen und anhand valider empirischer Daten die spezifischen Herausforderungen $\mathrm{zu}$ analysieren sowie daraus resultierende Bedürfnisse und Handlungsfelder aufzuzeigen. Es manifestierte sich dringlicher Forschungsbedarf darüber, wie Menschen, die in den letzten Jahren geflüchtet sind, mit dieser Krise im Kontext der Bedingungen der Aufnahmegesellschaften umgehen (vgl. WALKER 18-03-2020).

Am Ende des dritten Lockdowns (30. April 2021) hat der Public-Health-Experte Martin Sprenger ${ }^{1}$ kritisiert, dass die soziale Dimension der Pandemie viel zu wenig beachtet wurde. Die Pandemie hat gesundheitliche und soziale Ungleichheiten in der Gesellschaft vergrößert. Soziale Ungleichheit fördert nicht nur unterschiedliche Infektionsrisiken, sondern auch eine erhebliche Bandbreite an Erkrankungsrisiken. Sprenger verweist darauf, dass die Maßnahmen gegen die Pandemie Nachteile mit sich ziehen, die soziale Ungleichheiten zusätzlich weiter fördern. So sind ärmere Menschen eher von Wohnungslosigkeit betroffen, leiden stärker unter Arbeitsplatzverlust und haben kaum finanzielle Ressourcen, auf die sie zurückgreifen können.

Der Fokus im ersten Jahr der Pandemiebekämpfung lag nicht in einer Gesundheits-, sondern in einer Krankheitsorientierung, die zu einer Pathologisierung der Gesellschaft führte. Für die Gesundheitsorientierung sind soziale Beziehungen und Zusammenhalt evident. Martin SPRENGER vermerkt auch, dass Lehren aus dem Krisengeschehen gezogen werden müssen, um im medizinischen, sozialen und psychologischen Bereich darauf adäquat reagieren zu können.

Das Projekt möchte dazu beitragen, diese eingangs erwähnte Forschungslücke zu schließen und zugleich angesichts des Fortdauerns der Pandemie praxisbezogene und umsetzungsrelevante Empfehlungen an NGOs und politische Entscheidungsträger zu verbalisieren. Darüber hinaus bestand eine Intention darin, die Communities stärker und expliziter in die auf die Pandemie bezogenen Gegenmaßnahmen einzubeziehen und dies auch über die Projektlaufzeit hinaus. Die im Projekt hinzugewonnenen Daten sollen auch dazu verhelfen, Ungleichheiten und Ungleichgewichte im Integrations-

$<$ https://orf.at/stories/3211006/>. 
prozess zu verringern. Künftige Integrationskonzepte sollten die vielfältigen Auswirkungen der Krise auf Geflüchtete als besonders vulnerable Gruppen berücksichtigen sowie mögliche Entwicklungen im weiteren Verlauf der Krise (und eventuell nach deren Bewältigung) einbeziehen.

Eine wichtige Rolle im Rahmen der Analyse und Bewertung der Auswirkungen der Pandemie auf Individuen und Gruppen mit Fluchthintergrund spielt die Frage nach spezifischer Vulnerabilität. Die Intersektionalität von Flucht- bzw. Rechtsstatus (als Asylberechtige oder subsidiär Schutzberechtigte), von Diskriminierung und Xenophobie, von strukturellen Bedingungen und sozioökonomischem Status sowie ethnischer und religiöser Zugehörigkeit bedingt Vulnerabilitäten, die wenig sichtbar sind. Zusätzlich wirkt die allgemein prekäre Situation, die in hohem Ausmaß durch die unzureichende ökonomische Integration determiniert ist (DEMPSTER et al. 2020). Viele Betroffene sind Empfänger von AMS-Leistungen oder der bedarfsorientierten Mindestsicherung (MüLLER 10-08-2016) und oft in Segmenten des Arbeitsmarktes mit geringem Lohnniveau beschäftigt. Sie zählen zu einer diskriminierten Bevölkerungsgruppe, die vor allem im Falle der Afghan/inn/en - in der medialen Berichterstattung regelrecht stigmatisiert wird (ORTNER 02-05-2019). Hinzu treten komplexe Herausforderungen, die mit den Zugangsschranken zum kommunalen Wohnsektor (ZsCHIEDRICH 2016) in Wien sowie Informationsdefiziten hinsichtlich der Nutzung des staatlichen Gesundheitssystems generell und lokaler Gesundheitsversorgungsangebote zusammenhängen (RICHARDSON \& NORRIS 2010).

\section{VULNERABILITÄT UND VULNERABLE GRUPPEN}

Das Forscherteam hat versucht die multiplen Prozesse und Auswirkungen der Krise mit dem Vulnerabilitätsansatz zu betrachten. Es ist naheliegend anzunehmen, dass die Vulnerabilität der Asylwerber/inn/en, Asylberechtigten und subsidiär Schutzberechtigten aus Afghanistan und Syrien seit März 2020 beträchtlich zugenommen hat. Auf welche Weise und in welchem Ausmaß sich diese Veränderungen vollzogen und welche Konsequenzen daraus resultierten, zählte zu den Basisfragen des Forschungsprojekts.

Obwohl Vulnerabilität ein in der Fluchtforschung häufig verwendeter Begriff ist, existiert nach wie vor keine einheitliche oder allgemein akzeptierte Definition davon und auch die Frage, welche Gruppen als vulnerabel zu klassifizieren sind, bleibt umstritten (HruschKa \& Leboeuf 2019, p. 2; AtaK et al. 2018, p. 1 f.; Lorenz 2018; Brown et al. 2017, p. 497; ChAmbers 2006, p. 33). Die Konzeptualisierungen von Vulnerabilität von Hoffmaster (2006), MACKenziE et al. (2014a, b), AtaK et al. (2018) und LORENZ (2018) wurden von uns in einen Bezug zum konkreten Forschungskontext gestellt. Basierend auf den empirischen Ergebnissen werden auch die Interdependenzen zwischen der , inherent ${ }^{66}$ und ,situational vulnerability“ (MACKENZIE et al. 2014a b, p. 7) analysiert. Die ,situationale“6 (MACKENZIE et al. 2014a, p. 7; ATAK et al. 2018, p. 
3; LoRENZ 2018) und ,individuelle Vulnerabilität" (AIDA 2019, p. 9; UNHCR, June 2017) repräsentieren dabei zwei weitere Facetten. Unter Berücksichtigung der kritischen Diskurse über das Konzept im Allgemeinen und die uneinheitliche Terminologie im Zusammenhang damit (BANKoff 2001, 2007; ATAK et al. 2018; Brown et al. 2017; Chambers 1989, 2006; Stewart 2005; Mackenzie et al. 2014; Yaghmaian 2016) ist die Vulnerabilität mit Negativeffekten und Benachteiligungen zu verlinken, welche durch die Pandemiekrise hervorgerufen wurden und die die Geflüchteten auf ihrem Weg zur sozialen und Systemintegration (HeITMEYER \& IMBUSCH 2005; EsSER 1980, 2001) nachhaltig beeinträchtigen. Geflüchtete befinden sich aus vielerlei einander überschneidenden und interdependenten Ursachen in vulnerablen Situationen, sodass man in ihrem Fall von einer „Multivulnerabilität ${ }^{62}$ sprechen kann.

Eine der Ausgangshypothesen bezog sich darauf, dass komplexe Interdependenzen zwischen der inhärenten Vulnerabilität der Geflüchteten, den pandemiebezogenen politischen Maßnahmen, deren praktischen Auswirkungen und den wichtigen Bereichen der sozialen Inklusion und Systemintegration (Bildungssystem, Arbeits- und Wohnungsmarkt) bestehen. Diese Bereiche sind in divergierendem Ausmaß von den negativen und individuell belastenden Auswirkungen der Pandemie und der wirtschaftlichen Krise betroffen. Die Auswirkungen beziehen sich auf den Wohnsektor (Hang 2020; Iveson 2020; Rogers \& Power 2020), die Integration in den Arbeitsmarkt (Dempster et al. 2020; ILO June 2020, p. 2 ff.; March 2020; NASR 30-07-2020; Peters et al. 2020; World BANK Group 19-06-2020) sowie auf eine erhebliche Bandbreite an gesundheitsbezogenen Aspekten (BATALOvA \& FIX 2020; IMREF 2020). Etliche der auftretenden Probleme existierten zwar bereits vor Beginn der Pandemie (ANTEH 2019; Asgary \& Segar 2011; Menzel 2017) wurden durch diese aber modifiziert und erheblich verschärft. Beratungsangebote der NGOs, die Teilnahme an Ausbildungs- und Trainingsmaßnahmen, Deutschkurse sowie medizinische und psychotherapeutische Therapieangebote wurden stark beeinträchtigt, waren in der Regel nur mehr online möglich oder wurden sogar zur Gänze gecancelt. Sogar ein Anstieg der Diskriminierung von Geflüchteten war festzustellen (COATES 2020; DEvakumar et al. 2020; Logie et al. 2020).

Die inhärente und situationale Vulnerabilität von Geflüchteten lässt sie aus einer oberflächlichen Perspektive zunächst als eine weitgehend homogene Gruppe erscheinen, de facto stellen Geflüchtete aus Afghanistan und Syrien aber eine ethnisch, konfessionell, soziodemographisch, sozioökonomisch und bildungsmäßig überaus heterogene Gruppe dar (Kohlbacher et al. 2017, 2020; Kohlbacher \& Six-Hohenbalken 2019). Die spezifischen Krisenbewältigungsstrategien variieren je nach Geschlechtszugehörigkeit, Alterskohorte, Bildungsniveau, Familienstrukturen, dem rechtlichen Status etc. Die

2 Vgl. $<$ https://www.google.com/search?q=Multivulnerabilit\%C3\%A4t\&client=firefox-b$\mathrm{d} \&$ biw $=1920 \& \mathrm{bih}=938 \& \mathrm{tbm}=\mathrm{isch} \&$ source $=\mathrm{iu} \& \mathrm{ictx}=1 \&$ fir $=4 \mathrm{j} 9 \mathrm{sUyU} 4 \mathrm{PAiWXM} \% 252 \mathrm{ChdK}-$ KHjiziqMMM\%252C_\&vet=1\&usg=AI4_kQ576KTmRCHXeTwWMy5cDC8Xn0fZA\&sa= X\&ved=2ahUKEwjOzI7c9ZLsAhVy8uAK HUxEA1YQ9QF6BAgKEAo\#imgrc=4j9sUyU4P AiWXM>. 
SARS-CoV2-bezogenen Maßnahmen zeigen aber auch je nach Subgruppe innerhalb der Betroffenen divergierende Effekte, da Geschlechtszugehörigkeit, Bildungsniveau und rechtlicher Status die individuelle Situation jedes/r Geflüchteten entscheidend prägen. In Abhängigkeit von dieser Heterogenität bestehen auch unterschiedliche Risiken und ein divergierendes Ausmaß an Compliance bezüglich präventiver Maßnahmen der Regierung, wobei dies bislang nur Vermutungen waren und verlässliche empirische Daten weitgehend fehlten.

Autor/inn/en unterschiedlicher Disziplinen gehen davon aus, dass Menschen, die eine Flucht hinter sich haben, vulnerabel per se seien (AGIER 2011, p. 158; CHAmbers 2006, p. 33). Auch nach dem Erhalt eines positiven Asylbescheids verbleiben Menschen mit Fluchthintergrund in einem Zustand höchster Vulnerabilität, da die Einbindung in die unterschiedlichen gesellschaftlichen Bereiche der Residenzgesellschaften oft mit $\mathrm{zu}$ vielen Herausforderungen und Hürden verknüpft sind und somit auch nicht linear verlaufen können. Rückschläge, wiederkehrende Herausforderungen und die Intersektionalität von Problembereichen haben die Operation mit dem Konzept der Vulnerabilität zu einem wichtigen theoretischen Anknüpfungspunkt gemacht. Es handelt sich um eine Determinante von zentraler Wichtigkeit für die Charakterisierung und Bewertung der Auswirkungen von SARS-CoV2 und einen ,... entry point for discussing inequality or adversities of some kind“" (BROWN et al. 2017, p. 497).

Überlegungen zur Intersektionalität der vulnerabilitätserzeugenden Variablen seien beispielhaft kurz angeführt. Seit Beginn der Pandemie werden weltweit vor allem Personen im Alter von über 65 Jahren zu den Hochrisikogruppen gezählt. In der im österreichischen Immigrationskontext rezenten und demographisch jungen afghanischen Community ist diese Altersgruppe allerdings schwach vertreten. Ein höherer Anteil älterer Geflüchteter ist unter den Syrer/inne/n zu finden. Des Weiteren werden Frauen generell, Personen mit Traumatisierungen und bildungsferne Personen als spezifisch vulnerabel beziehungsweise als multivulnerabel angesehen. Eine große Gruppe innerhalb der afghanischen Community bilden die UMFs, wobei ehemalige und inzwischen bereits volljährig gewordene UMFs ebenfalls zu berücksichtigen sind.

Bereits existente Untersuchungen (DEMPSTER et al. 2020; ENDALE et al. 2020; PARVEen 18-05-2020; SAMAdDAR 2020; VOLKIN 20-04-2020; WALKer 18-03-2020) belegen, dass die Kausalfaktoren der Vulnerabilität der Geflüchteten stark verlinkt sind mit den Auswirkungen der Pandemie und vielfältige Faktorenkombinationen im Kontext des Gesundheitswesens inkludieren. Dazu zählen etwa der begrenzte oder schwierige Zugang zu präventivmedizinischer Versorgung (GUADAGNO 2020), ein Problem, welches auch bereits vor der aktuellen Pandemie vorhanden war (NeLLums et al. 2018) oder auch noch keine ausreichende Erfahrung in der Interaktion im Gesundheitswesen und dem Gesundheitssystem im Residenzland.

Weiters beinhaltet die Vulnerabilität von Geflüchteten im Kontext der Pandemie vielfältige Kombinationen von Faktoren wie soziale, sprachliche, ökonomische und wohnsituationsbezogene Barrieren, traditionelle, kulturell determinierte Paktiken der 
Betreuung von Erkrankten und des Umgangs mit Erkrankungen, ein erhöhtes Infektionsrisiko infolge hoher Wohndichte und großfamiliärer Strukturen, die immense Wichtigkeit sozialer Netzwerke innerhalb der Communities und darüber hinaus sowie traditionelle Vorgaben um Lebensübergänge (Geburt, Hochzeit, Tod) zu bestreiten (vgl. ENDALE et al. 2020; FAWAD et al. 2020; GAGE 2020; KLUGE et al. 2020; KoHLBACHER et al. 2017; Lau et al. 2020; Morgan \& Rose 2020; Rees \& Fisher 2020; Salmani et al. 14-07-2020; Truman et al. 2009, p. 278 ff.; VAughan \& Tinker 2009, p. 328 ff.).

Social Distancing und soziale Isolation stellen wichtige infektionsrisikoreduzierende Maßnahmen dar. Diese hängen aber wesentlich davon ab, ob Betroffene auch Zugang zu ausreichend großen Wohnflächen haben (ADDENDum 28-04-2018; Hang 2020; Ren 2020; FAIR Wohnen 2020, 24-27; Wittrich \& Pichler 11-05-2020). Die Wohnsituationen, etwa Unterkünfte für Personen, die noch im Asylverfahren sind, oder die strukturellen Zwänge (Privatwohnungen auf dem teuren Wiener Wohnungsmarkt oder noch kein Zugang zum sozialen Wohnungsmarkt) machen ein Physical Distancing oft kaum möglich. Die Einhaltung von Quarantänemaßnahmen ist in solchen Wohnsettings besonders schwierig (AIGNER 2016, 2018) wie auch die Aufrechterhaltung von sozialen Kontakten. Aber auch staatlich verordnete Maßnahmen, z.B. das Homeschooling der Kinder (Gogolin 2020, p. 175 ff.; SCHOBER et al. 2020), verunmöglichen Physical Distancing. Zahlreiche Interdendenzen zwischen räumlichen Faktoren, dem Social bzw. Physical Distancing und quarantänebedingten Absonderungsmaßnahmen werden berichtet (Rogers \& Power 2020; United Nations Human Rights OfFice OF THE COMmissioner 18-03-2020; VienNA.at, 29-07-2020). Diese beeinflussen wechselseitig die SARS-CoV2-Prävention und deren soziale Folgewirkungen.

Neben zahlreichen anderen negativen Folgewirkungen ist global auch von einem Ansteigen der innerfamiliären Gewalt infolge von Stressfaktoren (Verlust des Arbeitsplatzes, finanzielle Schwierigkeiten, Mangel an Wohnfläche etc.) die Rede (BuLler et al. 2020; BBC NEWS 06-04-2020, Refuge 06-04-2020; WAGERS 2020; Towensend 12-04-2020; TAUB 06-04-2020; WHO 14-05-2020). Für Österreich sind in diesem Zusammenhang zwar allgemein Zahlen verfügbar ${ }^{3}$, diese sind aber nicht disaggregiert nach der nationalen Herkunft der Gewalttäter vorhanden.

\footnotetext{
$3 \mathrm{Vgl} .<$ https://kurier.at/chronik/oesterreich/corona-lockdown-anstieg-bei-haeuslicher-gewaltgeringer-als-befuerchtet/401038904>: ,Die Coronakrise hat in Österreich aus Sicht von Innenminister Karl Nehammer (ÖVP) und Frauenministerin Susanne Raab (ÖVP) zu keinem signifikanten Anstieg bei häuslicher Gewalt geführt. $\mathrm{Zu}$ diesem Schluss sind die beiden am Montag bei einer gemeinsamen Pressekonferenz in Wien gekommen. ... 886 Mal wurden im vergangenen Februar in Österreich Annäherungs- und Betretungsverbote gegen Gewalttäter verhängt. Im März waren es bereits 972, im Lockdown-Monat April erhöhten sich die Fallzahlen dann auf 1.081 - deutlich unter dem Wert, der zu befürchten war, wie Raab betonte. Im Juli waren es 1.085 Fälle, im August 1.055, im September bisher 507.“
} 


\section{FORSCHUNGSFRAGEN}

Die folgenden Forschungscluster und -fragen standen im Fokus der Analysen:

- Welche Ähnlichkeiten und Unterschiede manifestieren sich hinsichtlich der Krisenperzeption, des Krisenmanagements und der Erwartungen während der Krise?

- Welche Reaktionen lassen sich seitens der Geflüchteten nachweisen und welche Coping-Strategien wurden seitens der Betroffenen entwickelt?

- Welche Auswirkungen sind in den Bereichen der Systemintegration festzustellen, also im Bildungs- und Gesundheitswesen und auf dem Arbeitsmarkt?

- Auf welche Weise wurden die sozialen Netzwerke der befragten Geflüchteten durch den Lockdown und die Ausgangsbeschränkungen beeinflusst und wie veränderten sich diese nach Aufhebung des strengen Lockdowns?

○ In welchem Ausmaß und auf welche Weise konnten Organisationen und Vereine der Communities den Verlust sozialer Netzwerke kompensieren? Welche Schritte wurden von diesen gesetzt, um SARS-CoV2-relevante Informationen zu verbreiten?

- Auf welche Weise hat die Pandemie die sozialen Interaktionen im Allgemeinen beeinflusst und verändert? Wie wurden die intrafamiliären, intergenerationalen, die Intra-community-und die Interaktionen außerhalb der Communities verändert?

○ Welche kulturspezifischen Normen fördern oder behindern individuelle Maßnahmen der Infektionsprävention, wie etwa das Physical Distancing, die Meidung von Sozialkontakten etc.?

- Auf welche Weise waren die Informationsbeschaffungskanäle und -modi von den pandemiebezogenen Gegenmaßnahmen betroffen? Wie konnten Geflüchtete ohne oder mit erschwertem Zugang zu digitalen Medien, nicht alphabetisierte Menschen oder jene mit geringen Sprachkenntnissen bzw. niedrigem Bildungsgrad die Situation managen und Zugang zu relevanten Informationen erlangen?

- Welche Bereiche haben alters-, geschlechts- oder wohnsituationsbedingt besondere Herausforderungen bedeutet und waren intersektional gesehen die Auslöser von vielschichtigen Folgewirkungen?

- Inwieweit war auch in den afghanischen und syrischen Communities ein Anstieg der innerfamiliären Gewalt bzw. Gewalt gegen Frauen festzustellen und welche primären Kausalfaktoren waren hierfür verantwortlich?

○ Wie können die Erfordernisse der physischen Distanzierung und andere notwendige Maßnahmen in den konkreten religiösen, kulturellen und Alltagskontexten umsetzbar gemacht werden und auf welche Weise können die Organisationen der Communities hierzu einen Beitrag leisten? Welche Implikationen kommen diesen Maßnahmen mit einer längeren Dauer der Pandemie und aktuellen Krise zu? 


\section{Relevante ANALYSEKategorien}

Sozialkontakte repräsentieren im Kontext der SARS-CoV2-Pandemie sowie der darauf bezogenen Maßnahmen eine Kategorie von zentraler Wichtigkeit, um die Ausbreitungswege und die „Geographie“ von Clustern zu analysieren und nachvollziehbar zu machen. Dies betrifft eigentlich alle durch zwischenmenschliche Interaktionen übertragbaren Infektionen und deren ,,infection pathways“ (BIAN 2004; BIAN \& Liebner 2007; Kuebart \& Stabler 2020; Lipsitch et al. 2003; Yu et al. 2004; vaN WAGNER 2008). Aus diesem Grund stellt das komplexe Feld sozialer Interaktionen einen wichtigen Teilbereich der Analysen im Rahmen dieses Berichts dar. Obwohl die Propagierung eines strikten Social Distancing, bei welchem es sich de facto aber um ein Physical Distancing handelt, zu einem generell starken Anstieg der Kommunikation und Interaktion über diverse Social Media (CHEN et al. 2020) geführt hat, bleiben reale Sozialkontakte gerade für Geflüchtete in vielen Integrationsbereichen von entscheidender Relevanz (KoHLBACHER et al. 2017).

Soziale Interaktionen und der räumliche Faktor stehen zueinander in einer Wechselwirkung (CASEY 2008). Soziale Nähe zwischen Individuen erhöht die Wahrscheinlichkeit engererer persönlicher und häufiger Kontakte, was wiederum die Infektionswahrscheinlichkeit erhöht (LLOYD-SMith et al. 2005). Aufgrund von Netzwerkstrukturen verbreiten sich Infektionen niemals gleichmäßig über räumliche Einheiten (ALI \& KeIL 2008). Gemäß der „Geographie“ der infektiösen Erkankungen (MEADE \& EARICKSON 2010) resultieren Infektionen aus einem Zusammenspiel von menschlicher Interaktionen und Umweltaspekten (KeELER \& EMCH 2018). Gerade Personen, die in größeren Haushalten, in mehrere Generationen umfassenden Haushalten sowie in Wohngemeinschaften leben und noch dazu in urbanen Agglomerationen, sind besonders betroffen (AigneR 2018, p. 779 ff.; KoHLBACHER 2019, p. 143 ff.). Darüber hinaus sind die günstigeren Wohnmöglichkeiten oft in Bezirken mit hohen Bevölkerungsdichten lokalisiert und dadurch sind Wahrscheinlichkeiten und Raten der Übertragung relativ höher. Medien berichten über die höchsten Infektionsraten in Wiener Bezirken mit hohen Bebauungsund Wohndichten (KRUTZLER 20-08-2020). KEELER \& EMCH (2018) plädieren daher für einen relationalen Fokus des wissenschaftlichen Zugangs, der soziale Verhaltensmuster der Bevölkerung bzw. bestimmter Gruppen und deren Interaktionen mit der physischräumlichen Umwelt einbezieht.

Für die Konzeptualisierung der Verbreitung infektiöser Erkrankungen ist also das „social networking“ als wichtige Kenngröße zu analysieren, da diese persönlichen Kontakte die Basis von Infektionsclustern bilden (KuEBarT \& STABLER 2020, p. 486). Dies bedeutet aber zugleich, dass soziale Interaktionen und Netzwerke, die eine immense Rolle für die soziale und die Systemintegration von Immigrant/inn/en spielen (GURAK \& Caces 1992; Hurlbert et al. 2000; Ryan 2011, p. 711 ff.; Philips 2006) nun zugleich zu einer Diffusion von Infektionen über persönliche Beziehungen und soziale Bindungen beitragen (BIAN 2004). Ein epidemiologisch und integrationsbezogen fataler Konnex! 
Mit Blick auf Familien- und Freundschaftsnetzwerke, Kontakte im beruflichen Kontext, Freizeitaktivitäten und -netzwerke wird somit die Analyse der variierenden Netzwerkstrukturen in diesen unterschiedlichen Kontexten relevant (RYAN 2011; CASTELlS \& Cardoso 2005). Dies steht in Akkordanz zu Granovetters (1973, p. 1995) etablierter Theorie der „weak and strong ties“, die darauf aufbaut, das die Qualität sozialer Beziehungen eine wichtige Rolle spielt. Aus räumlicher Perspektive sind die verschiedenen Ebenen und das facettenreiche Ineinandergreifen der individuellen und gruppenspezifischen Teilnetzwerkstrukturen relevant (CASTELls 2000; BIAN \& LIEBNER 2007).

Der räumliche Faktor hat bislang eher wenig Interesse in der Forschung erfahren, obwohl ,place impacting is a facilitator of outbreaks“, wie KUEBART \& STABLER (2020, 485) feststellten. Die Interdependenzen zwischen dem Raum und den Menschen sind hochrelevant für das Verständnis der Übertragungswege von infektiösen Erkrankungen (Meade 1977; Rogers \& Power 2020, p. 178 ff.; Menzel 2017, p. 19 ff.). Frühere Studien über Afghan/inn/en in Österreich haben die Relevanz des räumlichen Faktors für die Arbeits- und Wohnungsmarktintegration vor Augen geführt, aber auch was die Chancen im Bildungssystem anbelangt (KoHLBACHER et al. 2020). Nun ist davon auszugehen, dass der räumliche Faktor vor allem in Form der Wohndichte und -verhältnisse im Kontext der Pandemie zusätzliche Bedeutung gewonnen hat.

Vorhandene Analysen zu Afghan/inn/en in Österreich (KoHLBACHER et al. 2020; Filzmeier \& Perlot 2017, p. 10 ff.) belegen, dass über die Untersuchung kultureller Traditionen und gruppenspezifischen Verhaltens Aufschlüsse über soziale Interaktionsmodi sowie Risikobereitschaft, -vermeidung und -management im Allgemeinen zu gewinnen sind. Daher ist eine Interpretation der Ergebnisse im Kontext kulturspezifischer Faktoren relevant, um deren Einfluss auf das Gesundheitsbewusstsein der Geflüchteten adäquat bewerten zu können. Viele Alltagspraktiken und soziale Umgangsformen werden zwar auch durch die Religion beeinflusst, aber viel mehr noch durch kulturspezifische Normen (EMADI 2005; Zia 2020).

Eine Intention des Projekts bestand auch darin, die Diversität der Problematiken in den von der Pandemie betroffenen Gruppen mit Fluchterfahrung herauszuarbeiten. Damit gemeint ist die Diversität spezifischer Subgruppen und deren Betroffenheit durch die Krisensituation. Geflüchtete Frauen sind generell einer Vielzahl von Faktoren ausgesetzt, die Vulnerabilitäten erzeugen. Zu den Mehrfachbelastungen verheirateter Frauen mit der Verpflichtung zur Führung des Familienhaushaltes und der Unterstützung ihrer Kinder beim Homelearning kommen noch Gefahren innerfamiliärer Gewalt in diesen Ausnahmesituationen. Unverheiratete bzw. geschiedene/getrennte und alleinerziehende Frauen sind vielfach auch von Arbeitslosigkeit und verschärfter ökonomischer Marginalisierung betroffen und erfahren innerhalb der eigenen Community und Familie oft weniger Akzeptanz. Zwar ist der Anteil nicht alphabetisierter Personen bzw. solcher nur mit Grundschulabschluss in der afghanischen Community bei beiden Geschlechtern überproportional hoch und auch beträchtlich höher als in der syrischen Herkunftsgruppe, dennoch sind Bildungsdefizite unter Frauen erheblich öfter vorhanden als bei Männern 
(Buber-Ennser et al. 2016). Für diesen Personenkreis ist der Zugang zu Informationen die gesundheitliche Prävention oder die raschen Veränderungen der politischen Maßnahmen betreffend besonders schwierig. Eine weitere multivulnerable Gruppe unter den Geflüchteten sind schwer traumatisierte oder chronisch kranke Personen (FAWAD et al. 2020).

Gesundheitsexpert/inn/en plädieren dafür, Frauen eine wichtige Rolle im Rahmen präventiver Maßnahmen sowie der Gegenstrategien gegen die Virusausbreitung zuzuordnen (HARMAn 2016; MARINDo 2017; SMITH 2019; UN WOMEN 2020a, b; WHO 14-05-2020). Nachteilig ist, dass wenig genderbezogene bzw. genderdisaggregierte Daten hinsichtlich der Rolle der Frauen in der aktuellen Krise verfügbar sind. Dies beeinflusst intelligente Entscheidungen und passgenaue Gegenstrategien sowie jede Art gendersensitiver Maßnahmen gegen die Pandemie (PAPP \& Hersh, 27-03-2020; SMITH 2019; UN WOMEN 2020a, b; WHO 14-05-2020).

\section{Methodische Zugänge Und AbläUfe}

Methodische Zugänge, welche ausschließlich auf Expert/inn/enbefragungen basieren, sind nach MinKLER \& WALLERSTEIN (2003, p. 3 ff.) nicht immer optimal geeignet, um die Perspektiven Betroffener zu erheben und diese in ihrer Vielfalt adäquat abzubilden. Aus diesem Grund bediente sich das Projekt eines aufeinander aufbauenden Mixed-Method-Ansatzes, der Community-Based-Participatory-Research (CBPR)-Elemente beinhaltet und zu einer Mehrschrittanalyse führte. Dadurch wurden die Zugänge der befragten Expert/inn/en mit relevanten Resultaten aus der Bottom-upPerspektive durch die Involvierung der betroffenen Communities kombiniert (CHRIsTOPHER et al. 2008; JACOB et al. 2011; LEWIS-BECK et al. 2004). Die Umsetzung des Community-Based-Participatory-Research-Ansatzes (CBPR) war somit ein essentieller Teil des Projektdesigns, wobei syrische und afghanische Vereine als wichtige Projektpartner einbezogen wurden (HACKER 2013, IsRAEL et al. 2012).

Eine Grundlage für die vorliegende Untersuchung bildete ein kompilierter (teil) standardisierter Fragebogen, der vom Forschungsteam mittels Rückkoppelungsschleifen seitens der Communities erstellt wurde. Dieser fokussierte auf soziodemographische Basisdaten, Herausforderungen und Probleme, Coping Strategien, Sozialkontakte (soziale Isolation, Konflikte bzw. Gewalt in Partnerschaft/Ehe, faktische Veränderungen des Sozialverhaltens wie Social Distancing etc.), Gesundheit (psychischer und physischer Gesundheitszustand, Nutzung von Gesundheits- und Infektionsschutzmaßnahmen etc.), diverse Herausforderungen und Probleme (Informationsbeschaffung, Auswirkungen auf die finanzielle sowie die Arbeits- und Wohnsituation, Einhaltung der von der Regierung gesetzten Verkehrsbeschränkungen etc.), Bedeutung der Religion, Rolle der Communities und Unterstützungsstrukturen durch migrantische Vereine und NGOs (Übersetzungsleistungen, Online-Beratungen, Umgang mit Isolation, Unterstützung beim Distance Learning), wirtschaftliche und finanzielle Probleme. 
Zur Gewinnung möglichst valider Ergebnisse sowie zur Vermeidung eines Erhebungsbias sollte die Online-Befragung muttersprachlich (Arabisch, Farsi/Dari) durchgeführt werden, sodass auch Ältere und Personen mit geringer Deutschkompetenz befragt werden konnten. Angestrebt wurde ein Sample von $n=100$, d.h. je 50 Interviews pro Herkunftsgruppe. Schließlich wurden in summa 132 Fragebögen in der OnlineBefragung befüllt. Von der syrischen Community stammten 54 (alle auf Arabisch beantwortet, davon 10 analog abgegeben), von der afghanischen Community 78 (davon 57 auf Deutsch und 21 auf Farsi-Dari), wovon alle digital ausgefüllt worden waren.

Anfangs war die Rücklaufquote eher gering, die Ursachen für dies Verzögerung in der Rücklaufquote waren vielfältig: die generell heikle Thematik COVID-19, die bei vielen Geflüchteten Zurückhaltung auslöst, eine bei Geflüchteten aus häufig belastenden Erfahrungen im Asylverfahren resultierende generelle Verunsicherung hinsichtlich des Umgang und der Ziele von Befragungen und des Ausfüllens von Fragebögen oder mangelnde Routine und Erfahrungen im Umgang mit Fragebögen. Einige Face-to-FaceInterviews (v.a. jüngere Altersgruppen und Gebildete) erfolgten in deutscher bzw. auf expliziten Wunsch auch in englischer Sprache.

Des Weiteren wurde ein Leitfaden erstellt, um die Erfahrungen von Expert/inn/en zu erheben. Dieser wurde je nach Aufgabenbereich des/r befragten Experten/in modifiziert und umfasste die Schwerpunkte soziale Problembereiche, gesundheitliche Herausforderungen, persönliche und institutionelle Unterstützungsangebote und -leistungen der entsprechenden NGOs, zur Verfügung gestellte Informationsquellen, Informationsnutzung durch die Geflüchteten, alternative Informationsangebote (TV, Online-Medien, Communities) finanzielle Probleme, Homeschooling und Umstieg auf Online-Medien, Probleme bei der Beschaffung und Finanzierung, Sprachkompetenzen/Sprachbarrieren.

Die Audioaufnahmen der qualitativen Interviews wurden transkribiert und die Auswertungen auf Basis qualitativer Analyseverfahren durchgeführt (vgl. KRUSE 2015). Die quantitativen Daten wurden mittels SPSS uni- und bivariat ausgewertet, ein Großteil davon ist in Diagramm- oder in tabellarischer Form dargestellt.

Im Analyseprozess bedeutsam war die Vernetzung mit weiteren Forschungsprojekten, die gleichzeitig vom WWTF gefördert wurden. Hierbei war die Arbeitsgruppe „Vulnerable Gruppen“ mit ihren Vernetzungstreffen und Diskussionen über Vulnerabilität mehrfach gewinnbringend. Die Zusammenschau von verschiedenen vulnerablen Bevölkerungsgruppen (Hochrisikopatienten, Personen, die aufgrund ihres Alters oder ihrer psychischen Erkrankung in Einrichtungen untergebracht sind oder von NGOs intensiv betreut werden, und Geflüchtete) wirft nicht alleine neue theoretische und methodologische Fragestellungen auf, sondern auch neue Aspekte in der anwendungsorientierten Forschung. 


\section{AFGHAN/INN/EN UND SYRER/INNEN IN ÖSTERREICH - DIE UNTERSUCHTEN „COMMUNITIES“}

Der Begriff Communities bezieht sich in weiterer Folge auf einzelne Vereine, aber auch soziale Netzwerke und im weitesten Sinn auf das Herkunftsland. Der Begriff wird i. F. im Plural verwendet, um nicht den Eindruck einer Homogenisierung dieser sehr unterschiedlichen und diversen Gesellschaften zu erwecken.

Manche der Vereine, mit denen wir oder über die wir berichten, sind bereits vor den Fluchtbewegungen von 2015 entstanden, andere sind im Zusammenhang damit gegründet worden. Eine intensive Zusammenarbeit für die Fragebogenerstellung, die Online-Befragung und auch für einzelne Expert/inn/eninterviews erfolgte mit den Vereinen AKIS-EU (Afghanischer Kulturverein), „NEUER START“, mit dem „Österreichisch-arabischen Haus für Kunst und Kultur“, „Lifemakers“ und „Amal“.

\section{Geflüchtete aus Afghanistan}

Verglichen mit Deutschland oder Großbritannien, den großen Zielländern der Migrationsbewegung von Afghan/inn/en vor dem Krieg, kamen erst ab den späten 1960er-Jahren sukzessive und vorwiegend Männer nach Österreich. Primäres Ziel der vorwiegend aus dem urbanen Umfeld Stammenden war es ein Studium abzuschließen. Generell kann man von vier große Fluchtwellen aus Afghanistan nach Österreich sprechen. Die erste erfolgte zwischen dem kommunistischen Aufstand bis zum Sieg der Mujaheddin (1978-1992), die zweite während der Herrschaft der Mujaheddin (1992-1996), die dritte während der Herrschaft der Taliban (1996-2001) und die vierte in der Post-Taliban-Ära. Nach einem sukzessiven Anstieg der Asylanträge war 2015 ein Höhepunkt mit über 25.000 Asylwerber/inne/n zu verzeichnen. Seitdem ist die Zahl der Anträge stark rückläufig. Während bei früheren Fluchtbewegungen vor allem hochgebildete, wohlhabende und aus dem urbanen Milieu stammende Afghan/inne/n nach Österreich kamen (vgl. Alam 2008; KoHLBACHER et al. 2020), änderte sich dies im letzten Jahrzehnt. Nun sind sowohl gut ausgebildete als auch Personen, die aufgrund der jahrzehntelangen Kriegssituation keine oder kaum eine Ausbildung erhalten haben, nach Österreich gekommen. Auch änderte sich der ethnolinguistische und religiöse Hintergrund. Neben Paschtun/inn/en und Tadschik/inn/en waren nun auch Angehörige der demographisch kleineren Ethnien wie Usbek/inn/en und Hazara zunehmend unter den Geflüchteten (PotKANSKI 2011).

Bezüglich der Verteilung nach Alter und Geschlecht lässt sich ein extrem hoher Anteil an Männern (70,1\%) unter den in Afghanistan Geborenen erkennen. Die Altersstruktur der Diaspora wird von jüngeren und mittleren Alterskohorten dominiert. Der Anteil der kleineren Kinder ist eher gering: 5,3\% im Alter bis 6 Jahre. Aufgrund fehlender Studien über die transnationalen Netzwerke können nur grobe Schätzungen unternommen werden. Netzwerke richten sich nach den Herkunftsregionen und der 
Lokalisation von bestimmten Großfamilien in europäischen Ländern (v.a. Deutschland, Niederlande, Schweden) und Nordamerika.

Im letzten Jahrzehnt weist die Flucht von Afghan/inn/en nach Europa eine erhebliche Heterogenität in Bezug auf den ethnischen, politischen, sozioökonomischen und bildungsmäßigen Hintergrund auf. Auch die Vereinslandschaft hat sich diversifiziert und fokussiert nun u.a. auch auf die jüngere Generation und auf den Bildungsbereich.

\section{Geflüchtete aus Syrien}

Zwei Jahre nach Beginn des Krieges in Syrien (2011) war beinahe eine Million Syrer/innen auf der Flucht. Nach den Angaben des BundeSMINISTERIUMS Für INNERES waren ,2012 915 Personen, 2013 1.991, 2014 7.754, 201524.538 und 20168.845 SyrerInnen in Österreich angekommen (..." “. ${ }^{4}$ Mit der Intensivierung des Krieges 2015 stieg die Zahl der Asylanträge in Österreich sprunghaft an. Bezugnehmend auf das Herkunftsland von Asylsuchenden in den letzten fünf Jahren wurden die meisten Anträge von Syrer/inne/n gestellt. Die Zahl der Anträge hat in den letzten Jahren jedoch drastisch abgenommen. Im Jahr 2020 wurde 2.751 Personen aus Syrien Asyl und 318 subsidiärer Schutz gewährt, 2019 waren es 2.534, denen Asyl und 295, denen subsidiärer Schutz gewährt wurde. Im Jahr 2017 waren es 11.827 (Asyl) und 1194 (subsidiärer Schutz) und 2016 erhielten 15.528 Personen Asyl und 558 subsidiären Schutz. ${ }^{5}$

2016 wurde festgestellt, dass nur ein Drittel direkt aus Syrien nach Österreich kam, mehr als die Hälfte flüchteten über die Türkei. ${ }^{6}$ Die Geflüchteten aus Syrien haben einen hohen Bildungsstand vorzuweisen, mehr als die Hälfte hatte entweder eine Sekundarstufe (AHS, BHS) oder ein Universitätsstudium abgeschlossen. ${ }^{7}$

Bereits in den Jahrzehnten davor sind sukzessive Syrer/innen nach Österreich migriert, entweder aufgrund eines Studiums, wirtschaftlicher (Handel) oder politischer Gründe (Asylansuchen waren in den 1980er- und 1990er-Jahren eher vereinzelt gestellt worden). Die neu angekommenen Syrer/innen konnten in Wien und vereinzelt in den Landeshauptstädten syrische Communities vorfinden, die die ethnische und religiöse Struktur im Herkunftsland widerspiegelten.

Die Mehrheit der Syrer/innen sind sunnitische Muslime (73\%), gefolgt von der größten Minderheit den Alawiten (12\%). Etwa ein Zentel der Bevölkerung sind Christ/inn/en, die elf unterschiedlichen christlichen Konfessionen angehören (Syrischorthodox, maronitisch, armenisch - um nur einige zu nennen). Demographisch kleinere Religionsgemeinschaften sind die Drus/innen (4\%) und Schiit/inn/en (1\%). Mehr als ein Zentel der Bevölkerung sind Kurd/inn/en, von denen ein Großteil sunnitische Muslime sind. Unter den Kurd/inn/en werden auch die Yezid/inn/en gereiht. Zusätzlich zu den

\footnotetext{
$4 \quad$ BAUER et al. 2017, 118.

5 ASYlSTATISTIK ÖSTERREICH 2020, <https://www.migration-infografik.at/at-asylstatistiken2020/\#herkunftsstaaten>. 7. April 2021, Quelle: BMI.

6 BAUER et al. 2017, 105.

7 BubER-EnNSER et al. 2016, DiPAS.
} 
autochtonen religiösen und ethnischen Gruppen kommen noch palästinensische Flüchtlinge, die schon in den Jahrzehnten vor dem Kriegsausbruch 2011 nach Syrien geflüchtet waren (an die 480.000 Personen). ${ }^{8}$

Mangels genauer statistischer Angaben über die ethnischen oder religiösen Hintergründe der Geflüchteten können nur Grundlagenforschungen und Auftragsstudien einen ungefähren Einblick geben. In einer quantitativen Befragung von 2016 zeigte sich, dass unter den Geflüchtete nahezu alle Konfessionen, die es in Syrien gibt, vertreten waren. Manche dieser Konfessionen haben schon vor Jahrzehnten in Österreich Communities gegründet, so beispielsweise die syrisch-orthodoxe Kirche, die mittlerweile auch offiziell in Österreich anerkannt ist. Auch haben syrisch-katholische Christ/inn/en, Armenier/ innen, Assyrer/innen, Kurd/inn/en oder Yezid/inn/en schon seit einigen Jahrzehnten ihren Lebensmittelpunkt in Wien und hier Vereine, Einrichtungen, Organisationen und Netzwerke gegründet.

Syrer/innen, die in den letzten Jahren in Österreich um Asyl angesucht haben, trafen auf viele unterschiedliche jedoch damals noch demographisch kleinere politische, ethnische, religiöse und soziale Netzwerke und Strukturen. Syrer/innen und bereits die nachfolgende Generation waren als Akademiker/innen (u.a. Mediziner/innen), Wirtschaftstreibende, Künstler/innen oder als österreichische Politiker/innen tätig. Syrer/innen waren und sind Teil der arabischsprachigen Communities und Netzwerke, getragen von Personen aus unterschiedlichen Staaten des Nahen Ostens. Allen voran sind hier auch die Palästinenser/innen zu nennen, die bereits unter Bruno Kreisky besondere Förderung erfahren haben.

Diese Netzwerke haben gerade in den Anfangsjahren der Flucht enormes für die Integration geleistet, angefangen von der Unterstützung bei der Erstaufnahme, bei unterschiedlichen Beratungstätigkeiten und Integrationsunterstützung bei der Wohnungs- und Arbeitssuche. Vereine und Initiativen wurden gegründet, die sich beispielsweise um schulische, sprachliche oder soziale Belange der Neuangekommenen kümmerten. Bestehende diasporische Institutionen und Netzwerke haben sich auch in den letzten Jahren verändert und haben ihren Interessent/inn/en- bzw. Mitgliederkreis nicht nur zahlenmäßig erweitert. Von den Geflüchteten war auch bereits von Beginn an eine erhebliche Agency und Bereitschaft zur Selbstorganisation erkennbar. Vor allem spielten soziale Medien eine große Rolle.

$8 \quad$ Ibid., 111. 